\title{
Improving electrochemical properties by sodium doping for lithium-rich layered oxides
}

Kai Zhang ${ }^{\perp, \dagger}$ Hang Sheng, ${ }^{\perp, \dagger}$ Xiongwei $W u{ }^{\dagger}$ Lijun Fu, $\&$ Zhonghua Liu ${ }^{*}+$ Chunjiao Zhou,

${ }^{*},{ }^{\text {Rudolf Holze }}{ }^{ \pm}, \S$ and Yuping $W u,{ }^{*}, t, \mathcal{S}$

†School of Chemistry and Materials Science, Hunan Agricultural University, Changsha, 410128, China.

¥State Key Laboratory of Materials-oriented Chemical Engineering, School of Energy Science and Engineering, Nanjing Tech University, Nanjing, 211816, Jiangsu Province, China

§Institut für Chemie, AG Elektrochemie, Technische Universität Chemnitz, D-09107 Chemnitz,

Germany

*To whom correspondence should be addressed. E-mail: larkin-liu@163.com (Z.H.L); zcj69@163.com (C.J.Z); wuyp@fudan.edu.cn (Y.P.W) 


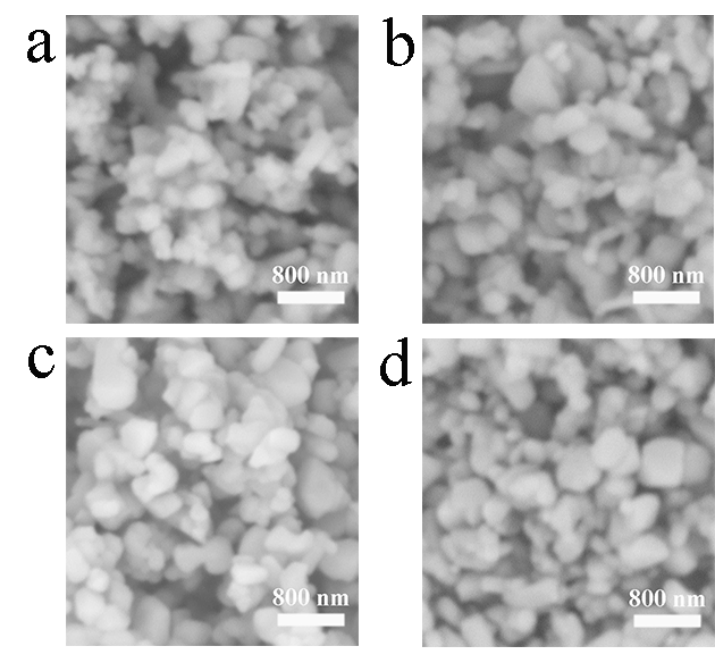

Figure S1. SEM images of the (a) LNMC, (b) $\mathrm{LNa}_{0.02} \mathrm{NMC}$, (c) $\mathrm{LNa}_{0.03} \mathrm{NMC}$ and (d) $\mathrm{LNa}_{0.04} \mathrm{NMC}$. 
Table S1 Comparison of the initial charge-discharge capacity and irreversible capacity.

\begin{tabular}{c|c|c|c}
\hline & $\begin{array}{c}\text { Charge } \\
\text { capacity } \\
\left(\mathrm{mAh} \cdot \mathrm{g}^{-1}\right)\end{array}$ & $\begin{array}{c}\text { Discharge } \\
\text { capacity } \\
\left(\mathrm{mAh} \cdot \mathrm{g}^{-1}\right)\end{array}$ & $\begin{array}{c}\text { Irreversible } \\
\text { capacity } \\
\left(\mathrm{mAh} \cdot \mathrm{g}^{-1}\right)\end{array}$ \\
\hline $\mathrm{LNMC}$ & 263.7 & 185.5 & 78.2 \\
$\mathrm{LNa}_{0.02} \mathrm{NMC}$ & 280.9 & 215.4 & 65.5 \\
$\mathrm{LNa}_{0.03} \mathrm{NMC}$ & 312.3 & 250.2 & 62.1 \\
$\mathrm{LNa}_{0.04} \mathrm{NMC}$ & 292.3 & 228.2 & 64.1 \\
\hline
\end{tabular}




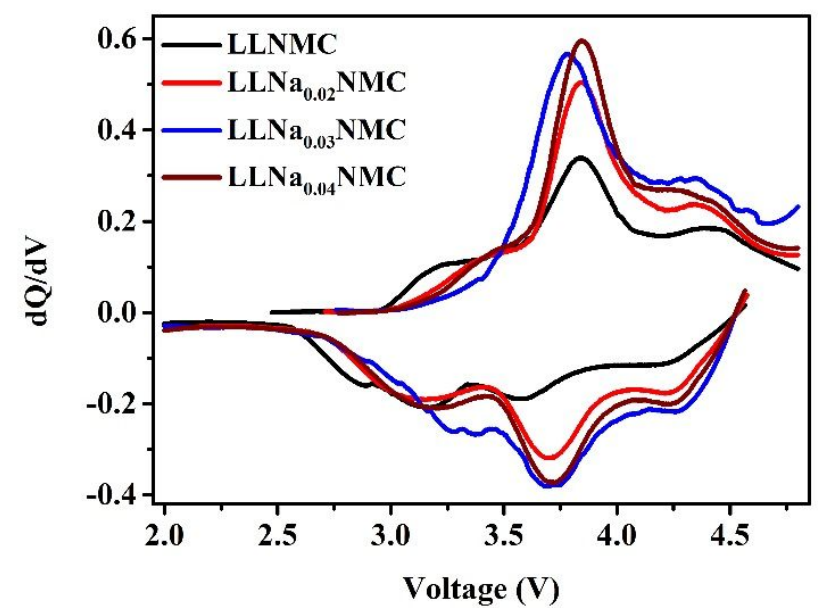

Figure S2. dQ/dV of LNMC and LNaxNMC after 50 rate performance cycle tests. 
Table S2 $\mathrm{R}_{\mathrm{ct}}$ of the LNMC and $\mathrm{LNa}_{\mathrm{x}} \mathrm{NMC}$ after the (a) 1 st and (b) 110th cycles.

\begin{tabular}{c|c|c}
\hline & 1 st & 100 th \\
\hline LNMC & $405.3 \Omega$ & $415.7 \Omega$ \\
$\mathrm{LNa}_{0.02} \mathrm{NMC}$ & $265.2 \Omega$ & $297.2 \Omega$ \\
$\mathrm{LNa}_{0.03} \mathrm{NMC}$ & $166.1 \Omega$ & $198.1 \Omega$ \\
$\mathrm{LNa}_{0.04} \mathrm{NMC}$ & $181.2 \Omega$ & $223.4 \Omega$ \\
\hline
\end{tabular}





Figure S3. Ex-situ XRD patterns of (a) LNMC and (b) $\mathrm{LNa}_{0.03} \mathrm{NMC}$ after 50th cycle. 